\title{
Component processes underlying future thinking
}

\author{
Arnaud D'Argembeau \\ University of Liège, Liège, Belgium \\ Claudia Ortoleva and Sabrina Jumentier \\ University of Geneva, Geneva, Switzerland \\ AND \\ MARTIAL VAN DER LINDEN \\ University of Liège, Liège, Belgium, \\ and University of Geneva, Geneva, Switzerland
}

\begin{abstract}
This study sought to investigate the component processes underlying the ability to imagine future events, using an individual-differences approach. Participants completed several tasks assessing different aspects of future thinking (i.e., fluency, specificity, amount of episodic details, phenomenology) and were also assessed with tasks and questionnaires measuring various component processes that have been hypothesized to support future thinking (i.e., executive processes, visual-spatial processing, relational memory processing, self-consciousness, and time perspective). The main results showed that executive processes were correlated with various measures of future thinking, whereas visual-spatial processing abilities and time perspective were specifically related to the number of sensory descriptions reported when specific future events were imagined. Furthermore, individual differences in self-consciousness predicted the subjective feeling of experiencing the imagined future events. These results suggest that future thinking involves a collection of processes that are related to different facets of future-event representation.
\end{abstract}

A remarkable feature of the human mind is its capacity to momentarily disengage from the immediate environment in order to contemplate hypothetical future scenarios (Suddendorf \& Corballis, 1997; Wheeler, Stuss, \& Tulving, 1997). This capacity to envision possible future events (hereafter, referred to as future thinking) has a strong adaptive value, allowing one, for example, to consider potential consequences prior to acting and, hence, to override current needs in favor of longer term goals (Boyer, 2008; Suddendorf \& Corballis, 2007; Tulving, 2005). Research has shown that future-oriented thoughts are pervasive in daily life (Klinger \& Cox, 1987) and serve important functions, such as planning, decision making, and emotion regulation (D'Argembeau, Renaud, \& Van der Linden, in press). In spite of this ubiquity and functional significance, the precise cognitive mechanisms underlying future thinking remain largely unexplored to date, although increased theoretical and empirical attention has been turned toward this issue in the past few years (e.g., Atance \& O'Neill, 2001; D'Argembeau \& Van der Linden, 2004; Gilbert \& Wilson, 2007; Schacter, Addis, \& Buckner, 2008; Suddendorf \& Corballis, 2007; Szpunar \& McDermott, 2008; Tulving, 2005). The purpose of the present study was to investigate the relative contributions of various component processes to the ability to imagine future events. Before detailing the specific aims of the study and the approach used, we first briefly review empirical data that have revealed that the imagination of future events critically depends on episodic memory, and we then present recently proposed theoretical hypotheses regarding the component processes involved in future thinking.

Evidence from various lines of research in psychology and cognitive neuroscience has now accumulated to indicate that remembering the past and imagining the future are intimately related (for recent reviews, see Schacter et al., 2008; Szpunar, 2010). First, there is evidence that individuals who present with episodic memory deficits have difficulties in imagining future events. This is most strikingly the case in amnesic patients (Hassabis, Kumaran, Vann, \& Maguire, 2007; Klein, Loftus, \& Kihlstrom, 2002; Tulving, 1985), but the relationship between episodic memory and future thinking abilities has also been observed in other populations, including older adults (Addis, Wong, \& Schacter, 2008), patients with Alzheimer's disease (Addis, Sacchetti, Ally, Budson, \& Schacter, 2009), depressed patients (Williams et al., 1996), and patients with schizophrenia (D'Argembeau, Raffard, \& Van der Linden, 2008). Developmental research has also revealed that episodic memory and future thinking emerge at the same time, between approximately 3 and 5 years of age (Atance \& Meltzoff, 2005; Suddendorf \& Busby, 2005). Furthermore, several functional neuroimaging

A. D’Argembeau, a.dargembeau@ulg.ac.be 
studies have shown that remembering past events and imagining future events rely on a common set of brain regions (Addis, Wong, \& Schacter, 2007; Botzung, Denkova, \& Manning, 2008; D’Argembeau, Xue, Lu, Van der Linden, \& Bechara, 2008; Okuda et al., 2003; Sharot, Riccardi, Raio, \& Phelps, 2007; Szpunar, Watson, \& McDermott, 2007). The phenomenological characteristics of autobiographical memories and the phenomenological characteristics of future event representations are affected similarly by a number of experimental manipulations (e.g., manipulations of emotional valence and temporal distance; D'Argembeau \& Van der Linden, 2004; Szpunar $\&$ McDermott, 2008), and individual differences in dimensions that are important for autobiographical memory retrieval (e.g., visual imagery ability) are similarly related to past and future events' characteristics (D'Argembeau \& Van der Linden, 2006). Research has also revealed that the temporal distribution of anticipated future events follows the same power function as the temporal distribution of remembered past events (Spreng \& Levine, 2006). Finally, there is evidence that errors in predicting future affective reactions relate to selective memory for past experiences (Gilbert \& Wilson, 2007).

These studies demonstrate the existence of a close relationship between remembering past events and imagining future events. The nature of this relationship is probably multifaceted. First, it has been suggested that mental representations of future events are constructed on the basis of various elements that have been extracted from past experiences (e.g., representations of objects, people, actions, locations, and emotions; Schacter \& Addis, 2007; Suddendorf \& Corballis, 1997, 2007). Episodic memory thus provides the necessary ingredients or vocabulary for constructing mental representations of the future. Second, remembering past events and imagining future events depend, to a large extent, on shared cognitive processes. Tulving $(1985,2005)$ is one of the first to have called attention to such commonalities, noting that "mental time travel" into the past and future involves a notion of self (the "traveler") and a sense of subjective time. More specifically, remembering past events and imagining future events require the capacity for self-consciousness and, in particular, the capacity to distinguish between self and others and between selves from distinct time periods (e.g., to understand that one's future self may have mental states that differ from one's current mental state; see also Klein, German, Cosmides, \& Gabriel, 2004; Suddendorf \& Corballis, 2007). Furthermore, remembering past events and imagining future events require the capacity to become aware of the temporal dimension of one's own experiences and involve processes that locate events along this subjective timeline (Klein et al., 2002; Suddendorf \& Corballis, 2007; Tulving, 2005).

Other authors have subsequently described a number of other key component processes (Hassabis \& Maguire, 2007; Schacter \& Addis, 2007; Suddendorf \& Corballis, 1997, 2007). Suddendorf and Corballis $(1997,2007)$ suggested that remembering past events and imagining future events require a processing space where information is temporarily maintained and manipulated (working memory), as well as processes that initiate, organize, and monitor the retrieval of relevant elements from long-term memory (executive processes) and processes that flexibly combine and integrate retrieved elements into a coherent representation (recursive and relational processes). Schacter and Addis (2007) focused on constructive and relational processes, emphasizing the importance of flexibly relating and recombining bits and pieces of information extracted from past episodes in order to create a coherent simulation of a new event that has not been experienced previously in the same form. Hassabis and Maguire made a related proposal and put special emphasis on visual-spatial processing, arguing that scene construction (i.e., the construction of a complex scene by retrieving relevant information and its integration into a coherent spatial context) is a key component process in supporting the remembering of past events and the imagining of future events. These views are consistent with the basic-systems model of autobiographical memory proposed by Rubin (2006), in which the remembering of past events (and, by extension, the imagination of future events) is supported by the interaction between systems that process modality-specific information, such as vision and other senses, spatial imagery, emotion, language, and narrative, as well as systems for retrieving and binding information from different systems. Finally, Buckner and Carroll (2007) called attention to self-projection processes, which enable the temporary withdrawal of attention from the immediate environment in order to mentally explore alternative perspectives.

Overall, then, it is likely that our ability to imagine or simulate future events relies on multiple component processes (including working memory, executive control processes, relational processes, visual-spatial processing, self-consciousness, and the apprehension of subjective time), most of which are also involved in remembering past episodes. The relative importance of different component processes, however, is still debated (cf. Buckner \& Carroll, 2007; Hassabis \& Maguire, 2007; Schacter et al., 2008) and requires further empirical investigation. In this study, we used an individual-differences approach to investigate this issue. The assumption behind this approach is that the relative contribution of different component processes to the imagination of future events can be assessed by examining the relationship between individual differences in the ability to imagine future events and individual differences concerning the processes thought to underlie such an ability. For example, insofar as executive processes contribute to the construction of future event representations, participants who score higher on measures of executive functioning should display better future-thinking abilities.

Following this logic, we investigated the relative contribution of different component processes to the imagination of future events. Specifically, participants completed a series of tasks and questionnaires measuring various component processes thought to underlie future thinking (i.e., working memory, executive functioning, visual-spatial processing, relational memory processing, self-consciousness, and time perspective), on the one hand, and several tasks assessing autobiographical memory and future thinking, on the other hand. Four kinds of measures that have been used in previous studies of future thinking were included in this 
study: a "future fluency task" (e.g., generating as many future events as possible for one minute; MacLeod \& Byrne, 1996; MacLeod \& Conway, 2007), which mostly measures the ability to access generic information regarding the future (e.g., abstract goals and plans, as well as extended or repeated events); a task assessing the ability to construct mental representations of specific future episodes (i.e., unique events that take place in a specific place at a specific time and that last a few minutes or hours, but not more than a day; Williams et al., 1996); a task assessing people's ability to generate episodic details once they have a specific event in mind (e.g., imagining precise sensory and contextual details, such as the color of an object or its location relative to other objects in the room; Hassabis et al., 2007); and rating scales that assess the phenomenological characteristics of mental representations of future events (e.g., the amount of visual details experienced, feeling of experiencing the event; D'Argembeau \& Van der Linden, 2004, 2006; Quoidbach, Hansenne, \& Mottet, 2008; Szpunar \& McDermott, 2008).

These different autobiographical measures probably tap predominantly different aspects of future thinking and should, therefore, show different relationships with at least some component processes. In this respect, we expected that executive processes involved in the organization and monitoring of retrieval would play a general role in future thinking and would thus be related to various measures of future thinking, whereas visual-spatial processing should show stronger relationships with tasks that assess the episodic dimension of future thinking (e.g., the amount of sensory descriptions referring to specific events). Furthermore, according to Klein et al. (2004), self-consciousness and the sense of temporality are involved in transforming information that is currently activated in the autobiographical database into an autobiographical experiencethat is, the feeling of mentally traveling through time to reexperience or preexperience events. Therefore, these dimensions should be particularly related to feelings of experiencing the imagined events.

Finally, in addition to examining relationships between measures of future thinking and measures of component processes, we were also interested in exploring possible differences between the component processes involved in remembering the past and imagining the future. For example, it has been suggested that constructive and relational processes are recruited to a greater extent when future events are imagined, as compared with when past events are remembered, because the simulation of future events requires that event details extracted from various past events be flexibly recombined into new associations (Schacter \& Addis, 2007). The present study allowed us to investigate that possibility.

\section{METHOD}

\section{Participants}

A total of 100 young adults ( 49 females), between 18 and 30 years of age ( $M=24.3$ years), took part in the study. ${ }^{1}$ They were all native French speakers, and their mean education was 15.2 years $(S D=$ 2.4). None of the participants had any history of neurological or psychiatric disorder.

\section{Materials}

The participants completed different measures of autobiographical memory and future thinking (i.e., fluency, specificity, amount of episodic details, and rating for phenomenological characteristics), a series of cognitive tasks measuring working memory, executive functioning, planning, visual-spatial processing, and relational memory processing, and two self-report questionnaires assessing self-consciousness and future time perspective.

Autobiographical fluency. The participants' ability in accessing/constructing generic representations of past and future events was assessed with autobiographical fluency tasks that were adapted from the work of MacLeod and colleagues (e.g., MacLeod \& Byrne, 1996). The participants were instructed to think about two time periods in the past (the last year and the last 5-10 years) and in the future (the next year and the next 5-10 years) and to generate as many events as possible that happened/might happen to them in the specified time period. The four time periods were presented orally, one at a time. Half of the participants received the two past time periods first, whereas the other half of the participants received the two future time periods first. Following MacLeod and Byrne, the 1 -year-distant time period was presented before the $5-10$ years time period, for both temporal directions. For each time period, the participants were given $60 \mathrm{sec}$ to generate as many events as possible. It was specified that the productions could refer to trivial or important events. No mention was made regarding event specificity.

Autobiographical specificity. The participants' ability to access/construct representations of specific past and future events was assessed with tasks adapted from the work of Williams et al. (1996). The participants were asked to recall specific events that happened in their personal past and to imagine specific events that might reasonably happen to them in the future in response to a series of cue words. They were told that the remembered/imagined events could be important or trivial but that they should be specific (i.e., unique events that take place in a specific place at a specific time and that last a few minutes or hours, but not more than a day). Some examples were provided to illustrate what would or would not be considered as a specific event, and practice trials were performed for both the past condition and the future condition. We selected cue words with various levels of imageability (i.e., words high in imageability, such as garden, and words low in imageability, such as choice) in order to maximize the sensitivity of the task in detecting individual differences in the ability to generate specific events in a nonclinical sample (previous studies have indeed shown that the imageability of cues correlates with the level of difficulty of the task in healthy young adults; Williams, Healy, \& Ellis, 1999). Two sets of five cue words were used (A and B), with the words in Sets A and B being matched for imageability $(M \mathrm{~s}=4.42$ vs. 4.28 , on a 7-point rating scale anchored at $1=$ low in imagery and $7=$ high in imagery; Desrochers \& Bergeron, 2000), frequency of use ( $M \mathrm{~s}=85.7$ vs. 89.8 per million of occurrence; New, Pallier, Brysbaert, \& Ferrand, 2004), and length (mean number of letters: $M \mathrm{~s}=6.6$ vs. 6.4 ). The assignment of Sets A and B to the past and future conditions was counterbalanced across participants. The order in which the participants completed the two conditions was also counterbalanced. In both conditions, the cues were written on cards that were presented one at a time. The participants were given $30 \mathrm{sec}$ to generate a specific event in response to each cue; if their first response was not a specific event, they were prompted again to recall or imagine a specific episode (e.g., "Can you think of a specific episode?").

Episodic details task. This task was adapted from Hassabis et al. (2007) and was used to investigate the amount of episodic details that participants were able to generate when recalling a specific past event and when imagining a specific future event. In the original task developed by Hassabis et al., participants were asked to vividly imagine atemporal scenes (e.g., "imagine you're lying on a white sandy beach in a beautiful tropical bay") and plausible future events (e.g., "imagine the next time you'll meet a friend"). In the present study, two cues were used, one cue for the future event condition (taken from Hassabis et al., 2007) and one additional cue 
created for the past event condition; the assignment of the two cues to the past and future conditions was counterbalanced across participants (i.e., "recall/imagine the last/next time you met/will meet a friend"; "recall/imagine something you did/will do during your last/ next vacation"). The participants were instructed to describe those events in as much detail as possible. It was specified that the events could refer to something trivial or important but that they should be specific (i.e., unique events that take place in a specific place at a specific time and that last a few minutes or hours, but not more than a day). For future events, it was further specified that the imagined events should be plausible (i.e., something they had planned or something that might reasonably happen) and new (i.e., something that did not occur in the past). There was no time constraint for this task, and, as in Hassabis et al., participants were instructed to "see the situation and setting in their mind's eye" as if they themselves were physically present and to describe as many sensory and introspective details about the situation as they could (i.e., what they see, hear, feel, and so forth). The order of presentation of the past versus future conditions was counterbalanced across participants. Immediately after having described each event, participants rated the phenomenological characteristics of their mental representation, using 7-point Likert-type rating scales (D'Argembeau \& Van der Linden, 2004, 2006). Mental representations of past and future events were assessed for amount of visual details (from $1=$ none to $7=a$ lot), clarity of location (from $1=$ not at all clear to $7=$ very clear), clarity of time of day (from $1=$ not at all clear to $7=$ very clear), feeling of emotion while remembering/imagining the event (from $1=$ none to $7=a$ lot), and feeling of mentally reexperiencing/ preexperiencing the event (from $1=$ not at all to $7=$ completely).

Letter-Number Sequencing subtest of the Wechsler Adult Intelligence Scale-III (WAIS-III; Wechsler, 2000). We used the Letter-Number Sequencing (LNS) subtest as a measure of working memory and controlled attention processes. This task is strongly correlated with laboratory working memory measures (e.g., operation span) and is a good predictor of fluid intelligence (Shelton, Elliott, Hill, Calamia, \& Gouvier, 2009). The experimenter reads mixed lists of digits and letters aloud to the participants, and they are asked to recall this list in correct numeric and alphabetic order. The complete test consisted of eight blocks with three trials in each. List length was three for the first block and increased by one for each successive block. Administration was terminated if participants missed all three trials within a block. The total number of correct trials was summed to create an LNS score (maximum possible score $=21$ ).

Verbal fluency tasks (VFTs). Participants were tested with validated French adaptations of phonemic and semantic fluency tasks (Cardebat, Doyon, Puel, Goulet, \& Joanette, 1990). In the phonemic task, participants were allowed 1 min to generate as many words as possible beginning with the letter P. In the semantic task, they had to generate as many names of animals as possible for $1 \mathrm{~min}$. Research suggests that phonemic and semantic fluency tasks impose comparable demands on executive processes involved in retrieval (i.e., initiation, efficient organization, and monitoring of retrieval) and are equally sensitive to frontal dysfunction (Henry \& Crawford, 2004).

Five-Point Test (Regard, Strauss, \& Knapp, 1982). The FivePoint Test (FPT) is a measure of figural fluency that has been developed as a nonverbal analogue to word fluency tasks. Participants are presented with a sheet of paper containing a series of dot matrices arranged in eight rows and five columns. The matrices are identical to the five-dot arrangement on dice. Participants are asked to produce as many different figures or designs as possible in $3 \mathrm{~min}$ by connecting the dots within each matrix. Only straight lines can be used, and all lines must connect dots. For each participant, we computed the total number of unique designs produced. Previous research has shown that the FPT is moderately correlated with verbal fluency, suggesting that verbal and nonverbal fluency measures tap similar, although not identical, functions (Regard et al., 1982). The FPT is also moderately correlated with visuospatial measures (e.g., block design).
Zoo Map Test (ZMT) from the Behavioural Assessment of Dysexecutive Syndrome battery (Wilson, Alderman, Burgess, Emslie, \& Evans, 1996). The ZMT is an ecological task that assesses the ability to formulate a sequence of operations intended for achieving some goal (i.e., planning). Participants are given a map of a zoo and have to plan a route to visit 6 of 12 locations, following certain rules. We used only the first ("high-demand") version of the test, which specifically assesses planning abilities. The score is the number of places visited in correct sequence minus the amount of errors.

Block Design Test (BDT) of the WAIS-III (Wechsler, 2000). This test is regarded as reflecting visuospatial constructive ability and is a reasonably good predictor of everyday spatial measures (Groth-Marnat \& Teal, 2000). It requires that a set of two-colored blocks are arranged so as to duplicate target patterns presented in order of ascending difficulty. The test was administered in a standard way, except that we started with Item 7 , which was completed successfully by all but 2 participants. The total raw score was computed for each participant (maximum possible score $=68$ ).

Visual Patterns Test (VPT; Della Sala, Gray, Baddeley, \& Wilson, 1997). The VPT is a measure of visual storage capacity in short-term memory. Participants are presented with matrix patterns of black and white squares in grids of varying size and are required to memorize a series of black-and-white checkerboard-like patterns of increasing complexity. We used Version A of the test and started at Level 8 of complexity. As was recommended by Della Sala et al. for use in scientific research, we computed for each participant the mean complexities of the last three patterns correctly recalled (maximum possible score $=15$ ).

Verbal Paired Associates I (VPA) subscale of the Wechsler Memory Scale-Third Edition (Wechsler, 2001). This task is one of the most widely used instruments for assessing relational episodic memory. Participants' task is to learn eight unrelated word pairs across four study-test trials. In this study, we used the recall score on Trial 1 (maximum possible score $=8$ ), instead of the total recall score, because the latter shows a performance ceiling effect in healthy young adults (Uttl, Graf, \& Richter, 2002).

Revised Self-Consciousness Scale (Scheier \& Carver, 1985; French adaptation by Pelletier \& Vallerand, 1990). The Revised Self-Consciousness Scale (RSCS) is a widely used 22-item questionnaire that has been developed to measure individual differences in three facets of self-consciousness: private self-consciousness, public self-consciousness, and social anxiety. In this study, we were interested only in the private self-consciousness dimension. The private self-consciousness subscale of the RSCS measures individual differences in the tendency to think about oneself and focus attention on one's inner experience (e.g., thoughts, feelings, physical sensations).

Zimbardo Time Perspective Inventory (Zimbardo \& Boyd, 1999; French adaptation by Apostolidis \& Fieulaine, 2004). The Zimbardo Time Perspective Inventory (ZTPI) measures individual differences in the perception of psychological time or time perspective. According to Zimbardo and Boyd, time perspective is the "process whereby the continual flows of personal and social experiences are assigned to temporal categories, or time frames, that help to give order, coherence, and meaning to those events" (p. 1271). The ZTPI measures individual differences in the tendency to emphasize and use different time frames: past, present, or future. The scale comprises five factors corresponding to distinct temporal orientations: past-negative, past-positive, present-hedonistic, present-fatalistic, and future. In this study, we were particularly interested in the future scale, which measures individual differences in the general tendency to anticipate the future and plan for future goals.

\section{Procedure}

The participants were tested individually in a quiet environment. After written informed consent was obtained, the participants completed the tasks in the following order: the VPA subscale, the VFTs, 
autobiographical fluency tasks, the LNS subtest, the ZMT, autobiographical specificity tasks, the BDT, the VPT, the episodic details task, the FPT, the RSCS, and the ZTPI. The three autobiographical tasks were presented in a fixed order due to the increasingly explicit instructions for the generation of episodic details. Half of the participants completed the past condition first, whereas the other half of the participants completed the future condition first. All responses provided in the three autobiographical tasks were audiorecorded and then retranscribed for scoring. The total testing time was about 90 min.

\section{Scoring of Responses Produced in Autobiographical Tasks}

All responses were first scored by one trained rater (C.O.). With regard to the autobiographical fluency task, the rater counted the number of events that were produced for each time period. General descriptions that did not refer to events (e.g., "I hope I'll have a happy life") were discarded. It was not required that a description refer to a specific event to count as a response, however (e.g., references to extended events, such as "I went on vacation to Spain," were allowed). Following MacLeod and Byrne (1996), we computed, for each participant, the total number of responses given for the two past time periods (past fluency score) and the total number of responses given for the two future time periods (future fluency score).

All responses given in the autobiographical specificity task were scored for specificity, following the criteria described by Williams and colleagues (e.g., Williams et al., 1996). A specific response referred to an event that happens at a particular time and place and does not last longer than a day (e.g., "I'm going to Barcelona for New Year's Eve. I imagine arriving at the hotel and having dinner with my friends in downtown Barcelona"). For each participant, we computed the total number of specific responses given in the past condition (past specificity score) and the total number of specific responses given in the future condition (future specificity score).

Finally, the descriptions given to the episodic details task were scored following criteria adapted from Hassabis et al. (2007). First, we scored the content of each description. The description was segmented into a set of statements, and each statement was classified into one of five content categories: spatial reference, entity, sensory description, thought/emotion/action, or temporal reference (the first four categories were the same as those in Hassabis et al., 2007, and the last category was added for the purpose of this study). The spatial reference category encompassed statements regarding the relative position of entities within the environment, directions relative to the participant's vantage point, or explicit measurements (e.g., "in front of the stage," "to my left there was," "about 200 meters from there"). The entity category was a simple count of how many distinct entities (e.g., objects, people, animals) were mentioned. The sensory descriptions category consisted of any statements describing (in any modality) properties of an entity (e.g., "he will be dressed in a dark suit"), as well as general weather and atmosphere descriptions (e.g., "it was very hot," "the room was very smoky"). The thought/ emotion/action category covered any introspective thoughts, emotional feelings, and actions of the participant (e.g., "I felt awkward"), as well as the thoughts, intentions, and actions of other entities in the scene (e.g., "my mother sat on a bench"). Finally, we added the temporal reference category to count statements providing a temporal context (e.g., "next summer") or time measurements (e.g., "we waited for two hours"). Repeated statements, irrelevant details, and other tangential information that could not be classified into one of these five categories were discarded. Following Hassabis et al., the score for each content category was capped at a maximum of 7 , and a total content score was computed by summing the scores of the five content categories (maximum possible total score $=35$ ). In addition to the total score, we also conducted analyses on the number of sensory descriptions, since sensory details are among the most important elements of episodic memories (Conway \& PleydellPearce, 2000; Rubin, 2006).

For each autobiographical task, a random selection of $20 \%$ of the responses was scored by a second independent rater (A.D.). There was good agreement between the two raters regarding the number of events produced in the autobiographical fluency task (intraclass correlation coefficient $[\mathrm{ICC}]=.85$ ), the number of specific events produced in the specificity task (ICC $=.93)$, and the number of elements generated for each content category in the episodic detail task (temporal references, ICC $=.93$; spatial references, ICC = .93; physical entities, ICC $=.92$; sensory descriptions, ICC $=.93$; thoughts/emotions/actions, ICC $=.75$ ).

\section{RESULTS}

Before examining the relationships between component processes and autobiographical measures, we first report descriptive statistics for the different measures included in this study, and we then describe exploratory principal components analyses (PCAs) that were carried out in order to investigate whether the data could be reduced before relationships between measures of component processes and autobiographical measures were looked at.

\section{Descriptive Statistics for Autobiographical Measures and Measures of Component Processes}

The means and standard deviations of the scores for the different autobiographical measures included in this study are shown in Table 1. As can be seen, scores for autobiographical fluency, autobiographical specificity, and amount of episodic details (total content score and sensory descriptions) were higher for past events than for

Table 1

Mean Performance on Autobiographical Tasks for Past and Future Events (With Standard Deviations)

\begin{tabular}{|c|c|c|c|c|c|}
\hline \multirow[b]{2}{*}{ Task } & \multicolumn{2}{|c|}{ Past Events } & \multicolumn{2}{|c|}{ Future Events } & \multirow[b]{2}{*}{$t(99)$} \\
\hline & $M$ & $S D$ & $M$ & $S D$ & \\
\hline Autobiographical fluency task & 17.05 & 6.11 & 15.33 & 5.70 & $3.18^{*}$ \\
\hline Autobiographical specificity task & 3.83 & 1.04 & 3.31 & 1.19 & $3.65^{*}$ \\
\hline \multicolumn{6}{|l|}{ Episodic Detail Task (Content) } \\
\hline Sensory descriptions & 5.13 & 2.06 & 4.52 & 2.21 & $2.67^{*}$ \\
\hline Total content score & 18.58 & 5.38 & 15.94 & 4.91 & $5.27^{*}$ \\
\hline \multicolumn{6}{|l|}{ Episodic Detail Task (Ratings) } \\
\hline Visual details & 5.66 & 1.36 & 5.36 & 1.39 & $2.04^{*}$ \\
\hline Clarity of location & 6.75 & 0.62 & 5.32 & 1.56 & $8.81^{*}$ \\
\hline Clarity of time of day & 6.44 & 1.02 & 4.73 & 1.91 & $8.22^{*}$ \\
\hline Feeling of emotion & 4.97 & 1.40 & 4.61 & 1.47 & 1.90 \\
\hline Feeling of experiencing & 5.05 & 1.45 & 4.93 & 1.39 & 0.77 \\
\hline
\end{tabular}


Table 2

Mean Performance (With Standard Deviation) and Factor Loadings for the Principal Components Analysis of the Eight Cognitive Tasks

\begin{tabular}{|c|c|c|c|c|c|}
\hline \multirow[b]{2}{*}{ Task } & \multicolumn{2}{|c|}{$\begin{array}{c}\text { Mean } \\
\text { Performance }\end{array}$} & \multirow{2}{*}{$\begin{array}{c}\text { Factor } 1 \\
\text { (Visual-Spatial } \\
\text { Processing) }\end{array}$} & \multirow{2}{*}{$\begin{array}{c}\text { Factor } 2 \\
\text { (Executive } \\
\text { Processes) }\end{array}$} & \multirow{2}{*}{$\begin{array}{r}\text { Factor } 3 \\
\text { (Verbal } \\
\text { Relationa } \\
\text { Memory) }\end{array}$} \\
\hline & $M$ & $S D$ & & & \\
\hline Letter-Number Sequencing & 11.78 & 2.62 & .36 & .34 & .48 \\
\hline Phonemic fluency & 15.00 & 4.20 & .19 & $.72^{*}$ & .04 \\
\hline Semantic fluency & 21.49 & 4.87 & -.13 & $.86^{*}$ & -.01 \\
\hline Five-Point Test & 35.07 & 8.67 & .44 & .47 & -.52 \\
\hline Zoo Map Test & 5.06 & 3.45 & .57 & -.15 & .26 \\
\hline Block Design Test & 48.93 & 12.89 & $.88^{*}$ & .17 & -.01 \\
\hline Visual Patterns Test & 9.58 & 1.80 & $.82^{*}$ & .12 & .13 \\
\hline Verbal Paired Associates & 2.49 & 2.10 & .19 & -.01 & $.81^{*}$ \\
\hline
\end{tabular}

${ }^{*}$ Highest factor loadings $(>.70)$.

future events (see Table 1 for $t$ values), which is in keeping with the results of earlier studies (e.g., Addis et al., 2008; D’Argembeau, Raffard, \& Van der Linden, 2008). Furthermore, ratings for phenomenological characteristics were higher for past events than for future events (for visual details, clarity of location, and clarity of time of day), which is also consistent with existing data (e.g., D'Argembeau \& Van der Linden, 2004, 2006). The means and standard deviations of the scores for the eight cognitive tasks included in this study are shown in Table 2. The mean score on the private self-consciousness subscale of the RSCS was $18.4(S D=4.8)$, and the mean score on the future scale of the ZTPI was $38.6(S D=6)$.

\section{PCAs}

We first carried out an exploratory PCA of the eight cognitive tasks to investigate whether the data could be reduced before relationships between measures of component processes and autobiographical measures were looked at. The PCA yielded three factors with eigenvalues greater than 1.0 , accounting for $64 \%$ of the total variance, and the scree plot indicated a clear "elbow" occurring after the third factor. Varimax rotation produced the factor pattern loadings shown in Table 2. The first factor can be considered as an index of visual-spatial processing; the BDT and VPT displayed high loadings on this factor, and the ZMT and FPT also had moderate loadings. The second factor can be considered as a measure of executive processes involved in the organization and monitoring of retrieval; both phonemic and semantic fluency tasks displayed high loadings on this factor, and the FPT also had a moderate loading. The third factor can be considered as representing verbal relational memory, the VPA being the only task displaying a high loading on this factor. The LNS subtest had moderate loadings on the three factors. For convenience, we will call these factors visual-spatial processing (Factor 1), executive processes (Factor 2), and verbal relational memory (Factor 3). Standardized factor scores for each of these three factors were computed for each participant and were then used in subsequent analyses.

We also carried out an exploratory PCA of the five rating scales for phenomenological characteristics. For past events, the PCA yielded two factors with eigenvalues greater than 1.0, accounting for $66 \%$ of the total variance. Varimax rotation produced the factor pattern loadings shown in Table 3. The first factor can be considered as an index of the subjective amount of episodic details; ratings for visual details, clarity of location, and clarity of time of day displayed high loadings on this factor. The second factor can be considered as a measure of feelings of experiencing the event; both ratings for feeling of reexperiencing and ratings for feeling emotions displayed high loadings on this factor. For convenience, we will call these factors episodic details (Factor 1) and feeling of experiencing (Factor 2). Standardized factor scores for each of these two factors were computed for each participant and were then used in subsequent analyses.

For future events, the PCA also yielded two factors, accounting for $61 \%$ of the total variance. Varimax rotation produced the factor pattern loadings shown in Table 3 . The

Table 3

Factor Loadings for the Principal Components Analysis of Ratings for Phenomenological Characteristics

\begin{tabular}{|c|c|c|c|c|}
\hline \multirow[b]{2}{*}{ Characteristic } & \multicolumn{2}{|c|}{ Past Events } & \multicolumn{2}{|c|}{ Future Events } \\
\hline & $\begin{array}{l}\text { Factor } 1 \\
\text { (Episodic } \\
\text { Details) }\end{array}$ & $\begin{array}{c}\text { Factor } 2 \\
\text { (Feeling of } \\
\text { Experiencing) }\end{array}$ & $\begin{array}{c}\text { Factor } 1 \\
\text { (Feeling of } \\
\text { Experiencing) }\end{array}$ & $\begin{array}{c}\text { Factor } 2 \\
\text { (Episodic } \\
\text { Details) }\end{array}$ \\
\hline Visual details & $.71^{*}$ & .14 & .15 & $.76^{*}$ \\
\hline Spatial context & $.74^{*}$ & .16 & .49 & .47 \\
\hline Temporal context & $.83^{*}$ & -.08 & .05 & .68 \\
\hline Feeling emotions & -.10 & $.88^{*}$ & $.90^{*}$ & -.03 \\
\hline Feeling of experiencing & .42 & $.72^{*}$ & $.80^{*}$ & .30 \\
\hline
\end{tabular}

${ }^{*}$ Highest factor loadings $(>.70)$. 
Table 4

Correlations Between Measures of Component Processes and Autobiographical Measures for Past Events

\begin{tabular}{|c|c|c|c|c|c|}
\hline Measure & $\begin{array}{c}\text { Visual-Spatial } \\
\text { Processing }\end{array}$ & $\begin{array}{l}\text { Executive } \\
\text { Processes }\end{array}$ & $\begin{array}{c}\text { Verbal } \\
\text { Relational } \\
\text { Memory }\end{array}$ & $\begin{array}{c}\text { Self- } \\
\text { Consciousness }\end{array}$ & $\begin{array}{c}\text { Future } \\
\text { Orientation }\end{array}$ \\
\hline Autobiographical fluency & $.09(-.11-.28)$ & $.25^{*}(.06-.43)$ & $-.12(-.31-.08)$ & $.03(-.17-.23)$ & $.12(-.08-.31)$ \\
\hline Autobiographical specificity & $.01(-.19-.21)$ & $.05(-.15-.24)$ & $-.09(-.28-.11)$ & $.08(-.12-.27)$ & $-.12(-.31-.08)$ \\
\hline \multicolumn{6}{|l|}{ Episodic Detail } \\
\hline Total content score & $-.03(-.23-.17)$ & $.28^{*}(.09-.45)$ & $-.12(-.31-.08)$ & $.03(-.17-.23)$ & $.12(-.08-.31)$ \\
\hline Sensory descriptions & $.05(-.15-.24)$ & $.22^{*}(.03-.40)$ & $-.03(-.23-.17)$ & $.09(-.11-.28)$ & $.04(-.16-.23)$ \\
\hline \multicolumn{6}{|c|}{ Phenomenological Characteristics } \\
\hline Episodic details & $.05(-.15-.24)$ & $.04(-.16-.23)$ & $.07(-.13-.26)$ & $.08(-.12-.27)$ & $.11(-.09-.30)$ \\
\hline Feeling of experiencing & $-.04(-.23-.16)$ & $-.05(-.24-.15)$ & $-.18(-.36-.02)$ & $-.01(-.21-.19)$ & $.09(-.11-.28)$ \\
\hline
\end{tabular}

Note $-95 \%$ confidence intervals are shown in parentheses. $\quad{ }^{*}$ Correlations significant at $p<.05$, corrected for multiple comparisons using the false discovery rate procedure.

Table 5

Correlations Between Measures of Component Processes and Autobiographical Measures for Future Events

\begin{tabular}{lccccc}
\hline \multicolumn{1}{c}{ Measure } & $\begin{array}{c}\text { Visual-Spatial } \\
\text { Processing }\end{array}$ & $\begin{array}{c}\text { Executive } \\
\text { Processes }\end{array}$ & $\begin{array}{c}\text { Verbal } \\
\text { Relational } \\
\text { Memory }\end{array}$ & $\begin{array}{c}\text { Self- } \\
\text { Consciousness }\end{array}$ & $\begin{array}{c}\text { Future } \\
\text { Orientation }\end{array}$ \\
\hline $\begin{array}{l}\text { Autobiographical fluency } \\
\text { Autobiographical specificity }\end{array}$ & $.01(-.19-.21)$ & $.20(.01-.38)$ & $-.17(-.35-.03)$ & $.15(-.05-.34)$ & $.10(-.10-.29)$ \\
Episodic Detail & $.06(-.14-.25)$ & $.23^{*}(.04-.41)$ & $.10(-.10-.29)$ & $-.07(-.26-.13)$ & $-.18(-.36-.02)$ \\
$\quad$ Total content score & $.12(-.08-.31)$ & $.33^{*}(.14-.49)$ & $-.02(-.22-.18)$ & $.18(-.02-.36)$ & $.13(-.07-.32)$ \\
$\quad \begin{array}{l}\text { Sensory descriptions } \\
\text { Phenomenological Characteristics }\end{array}$ & $.25^{*}(.06-.43)$ & $.32^{*}(.13-.49)$ & $.03(-.17-.23)$ & $.21^{*}(.02-.39)$ & $.25^{*}(.06-.43)$ \\
$\quad \begin{array}{l}\text { Episodic details } \\
\text { Feeling of experiencing }\end{array}$ & $-.01(-.21-.19)$ & $-.01(-.21-.19)$ & $-.01(-.21-.19)$ & $.15(-.05-.34)$ & $.10(-.10-.29)$ \\
Note-95\% confidence intervals are shown in parentheses. & ${ }^{*}$ Correlations significant at $p<.05$, corrected for multiple comparisons using \\
the false discovery rate procedure. & & & & $.16(-.04-.35)$
\end{tabular}

first factor can be considered as an index of feelings of experiencing the event; both ratings for feeling of experiencing and ratings for feeling emotions displayed high loadings on this factor. The second factor can be considered as a measure of the subjective amount of episodic details; ratings for visual details displayed high loadings on this factor, and ratings for spatial and temporal information displayed moderate loadings on this factor. For convenience, we will call these factors episodic details (Factor 2) and feeling of experiencing (Factor 1). Standardized factor scores for each of these two factors were computed for each participant and were then used in subsequent analyses.

\section{Correlations Between Autobiographical Measures and Measures of Component Processes}

The correlations between the different autobiographical measures and measures of component processes, along with their $95 \%$ confidence interval (CI), are shown in Table 4 for past events and in Table 5 for future events. A correlation was considered statistically significant if $p<.05$, corrected for multiple comparisons using the false discovery rate procedure (Benjamini \& Hochberg, 1995).

The first point to note is that there were significant correlations between executive processes and various autobiographical measures: with autobiographical fluency and measures of episodic details (total content score and sensory descriptions) for past events, and with auto- biographical specificity and measures of episodic details (total content score and sensory descriptions) for future events. The second point to note is that there was a significant correlation between visual-spatial processing and the number of sensory descriptions reported, but only for future events. We did not observe any significant correlations between autobiographical measures and verbal relational memory, either for past events or for future events. For future events, self-consciousness (as measured by the private self-consciousness subscale of the RSCS) showed significant correlations with sensory descriptions and with ratings of feeling of experiencing. Finally, we also observed a significant correlation between future orientation (as assessed by the future subscale of the ZTPI) and the number of sensory descriptions provided when future events were imagined. ${ }^{2}$

\section{Multiple Regression Analyses Predicting Autobiographical Measures for Future Events}

The correlation analyses reported above showed that several component processes (i.e., executive processes, visual-spatial processing, self-consciousness, and future orientation) were correlated with autobiographical measures for future events. To assess the independent contribution of each of these processes to the prediction of autobiographical measures, we entered all variables simultaneously into multiple regression analyses. The results are shown in Table 6 . These analyses showed that executive processes, visual-spatial processing, and 
Table 6

Multiple Regression Analyses Predicting Autobiographical Measures for Future Events

\begin{tabular}{|c|c|c|c|c|}
\hline \multirow[b]{2}{*}{ Dependent Variable } & \multicolumn{4}{|c|}{$\beta$ Weights for Independent Variables $(95 \% \mathrm{CI})$} \\
\hline & $\begin{array}{l}\text { Executive } \\
\text { Processes }\end{array}$ & $\begin{array}{c}\text { Visual-Spatial } \\
\text { Processing }\end{array}$ & $\begin{array}{c}\text { Self- } \\
\text { Consciousness }\end{array}$ & $\begin{array}{c}\text { Future } \\
\text { Orientation }\end{array}$ \\
\hline Autobiographical fluency & $.25^{*}(.05-.44)$ & $.09(-.11-.29)$ & $-.02(-.23-.18)$ & $.12(-.08-.32)$ \\
\hline Autobiographical specificity & $.23^{*}(.04-.43)$ & $.05(-.14-.25)$ & $-.03(-.23-.17)$ & $-.18(-.36-.02)$ \\
\hline \multicolumn{5}{|l|}{ Episodic Detail } \\
\hline Total content score & $.32 *(.14-.51)$ & $.11(-.07-.30)$ & $.14(-.06-.33)$ & $.09(-.11-.28)$ \\
\hline Sensory descriptions & $.30^{*}(.13-.48)$ & $.25^{*}(.07-.43)$ & $.12(-.06-.31)$ & $.22 *(.03-.40)$ \\
\hline \multicolumn{5}{|c|}{ Phenomenological Characteristics } \\
\hline Episodic details & $-.01(-.21-.19)$ & $-.02(-.22-.18)$ & $.14(-.07-.34)$ & $.06(-.15-.27)$ \\
\hline Feeling of experiencing & $.13(-.06-.32)$ & $.15(-.04-.34)$ & $.27^{*}(.07-.47)$ & $.10(-.13-.27)$ \\
\hline
\end{tabular}

${ }^{*} \beta$ weights significant at $p<.05$.

future orientation all contributed independently to the prediction of the amount of sensory descriptions reported when future events were imagined. The measure of executive processes was the only independent predictor of autobiographical fluency, autobiographical specificity, and total content score for episodic details. Finally, selfconsciousness contributed independently to the prediction of feelings of experiencing imagined future events.

\section{DISCUSSION}

Although increased theoretical and empirical attention has been turned toward future thinking in the past few years, the precise cognitive mechanisms that underpin our ability to imagine or simulate future events remain largely unexplored. In this study, we investigated this issue using an individual-differences approach. Participants completed several measures of autobiographical memory and future thinking (i.e., fluency, specificity, amount of episodic details, and ratings for phenomenological characteristics), as well as a series of tasks and questionnaires measuring various component processes that are thought to play key roles in future thinking (i.e., executive processes, visual-spatial processing, relational memory processing, self-consciousness, and time perspective). In addition to examining relationships between measures of future thinking and measures of component processes, we were also interested in exploring possible differences between the component processes involved in remembering past events and the component processes involved in imagining future events. The main results can be summarized as follows: (1) Measures of executive processes involved in the organization and monitoring of retrieval were correlated with several autobiographical measures (e.g., fluency, total amount of episodic details, amount of sensory descriptions), for both past events and future events; (2) measures of visual-spatial processing abilities were correlated specifically with the number of sensory descriptions reported, but only for future events; (3) both self-consciousness and future orientation were correlated with the number of sensory descriptions reported when future events were imagined, and self-consciousness also was correlated with ratings for feeling of experiencing the imagined events; (4) multiple regression analyses showed that executive processes, visual-spatial processing, and future orientation all contributed independently to the prediction of the number of sensory descriptions reported when future events were imagined.

The first point to consider is that measures of executive processes involved in the organization and monitoring of retrieval were correlated with various autobiographical measures (i.e., fluency, episodic details), for both past events and future events. These findings suggest that executive processes play a general role in accessing and representing autobiographical knowledge. Executive processes may support the strategic aspects of autobiographical knowledge retrieval (e.g., establishing retrieval goals, initiating and guiding search, monitoring and selecting retrieved information; Conway \& Pleydell-Pearce, 2000; Rubin, 2006), which certainly play a key role in both remembering past events and imagining future events, at least when there is a willful attempt to retrieve autobiographical knowledge (see Berntsen \& Jacobsen, 2008, however, for evidence that both autobiographical memories and representations of future events can be formed spontaneously and, thus, presumably with little involvement of executive processes). Measures of executive processes were also correlated with measures of event specificity, but only for future events. Constructing the representation of a specific event typically requires multiple search cycles in memory (as well as monitoring processes that verify the relevance of search output) until sufficient information is accessed for generating a unique event that can be located at a particular place and time (Williams et al., 1996). The present findings thus suggest that generating representations of unique episodes that are precisely located in space and time places higher demands on executive processes for the future than for the past. This might be so because, whereas a particular past event has been experienced in a unique spatiotemporal context (i.e., the event occurred in a specific place at a specific time), a future event can be imagined as happening in many different spatiotemporal contexts (e.g., meeting John in Paris next weekend vs. meeting John in New York a month from now). Additional executive processes may therefore be required for selecting a particular spatiotemporal context (among multiple possible contexts) that is plausible and coherent with other features of the simulated event (e.g., the persons involved).

We also found that the imagination of future events and, more specifically, the amount of sensory details generated 
was correlated with our index of visual-spatial processing abilities, whereas such a correlation was not observed for past events. Furthermore, results from regression analyses showed that visual-spatial processing abilities made unique contributions (in addition to executive processes and future orientation) to the prediction of the number of sensory details generated during the imagination of future events. These findings are important to consider because sensory details and, in particular, visual details are key elements of autobiographical memory and future thinking that provide event representations with "episodic flavor" (D’Argembeau \& Van der Linden, 2006; Rubin, Schrauf, $\&$ Greenburg, 2003). The task showing the highest factor loading on our index of visual-spatial processing was the BDT of the WAIS-III. When considering the demands of this task, one can only be struck by its similarity to the processes thought to be involved in constructing detailed representations of future events: Both require combining multiple elements (i.e., blocks for the BDT vs. details about objects, people, and so forth for future events) to form a coherent visuospatial whole. The present findings thus provide support for the idea that visual-spatial constructive processes play a key role in the ability to create mental representations of future events that are infused with many sensory details (Hassabis \& Maguire, 2007; Schacter \& Addis, 2007).

Our findings also suggest that remembering sensory details about past events places fewer demands on visualspatial constructive abilities than does imagining details of future events. This result is consistent with the view that imagining a specific future event engages more intensive constructive activity, relative to remembering a past event, because the individual has to flexibly recombine multiple details (e.g., details about objects, people, locations) to generate a novel episode that has not been experienced as such in the past (Schacter \& Addis, 2007, 2009). ${ }^{3}$ Neuropsychological and functional neuroimaging studies suggest that the medial temporal lobe plays a key role in underpinning the kind of constructive processes involved in generating detailed simulations of future events (Addis, Pan, Vu, Laiser, \& Schacter, 2009; Hassabis et al., 2007; Schacter \& Addis, 2009). Interestingly, there is also evidence that medial temporal lobe atrophy is associated with poor performance on the BDT (Jokinen et al., 2004), which is consistent with the idea that this task may tap, at least in part, into processes that are similar to those engaged during the imagination of future events.

Contrary to executive processes and visual-spatial processing, the measure of relational episodic memory processing that was included in the present study (i.e., the VPA of the Wechsler Memory Scale-Third Edition) was not correlated with the ability to generate future events (or with the ability to remember past events). A recent metaanalysis of functional neuroimaging studies has revealed that laboratory-based episodic memory tasks and autobiographical memory tasks engage largely nonoverlapping brain regions, suggesting that the two types of tasks depend, at least in part, on distinct processes (McDermott, Szpunar, \& Christ, 2009). It should be noted, however, that the present findings contrast with those of another recent study, in which performance on the VPA was positively correlated with the number of episodic details produced when past and future events were generated (Addis et al., 2008). This correlation was obtained in a sample of older adults, however, so the divergent findings between the two studies could be due to age-related changes in the component processes underlying future thinking, or it might simply be that the VPA is more sensitive in detecting individual differences in episodic relational memory in older adults than in younger adults. Additional research should therefore be conducted (using both young and older adult samples) before drawing any firm conclusion regarding the contribution of verbal relational memory to futurethinking abilities. More generally, it should be noted that the individual-differences approach used in the present study, as well as in other studies, involves averaging autobiographical measures from several individual events. Intraindividual variability in autobiographical measures might thus create noise in the analyses of individual differences, which might limit the sensitivity of this approach for detecting relationships with component processes. The present finding that some component processes were associated with measures of future thinking, whereas others were not, cannot be simply explained by this possibility, however.

Finally, it is also important to note that executive processes and visual-spatial constructive abilities were not the only component processes that were correlated with future thinking measures in the present study. In particular, we found that individual differences in future orientation (i.e., the general tendency to consider the future in order to organize and give meaning to one's experiences; Zimbardo \& Boyd, 1999) made an independent contribution to the prediction of the number of sensory details generated during the imagination of future events. Furthermore, selfconsciousness (more specifically, the general tendency to think about the self and to focus attention on one's inner experience; Scheier \& Carver, 1985) contributed to the prediction of feelings of experiencing when future events were imagined. These findings are consistent with the idea that the ability to represent one's own mental states and the ability to apprehend subjective time may be involved in transforming accessed autobiographical knowledge into the subjective experience of "traveling through time" to preexperience a particular future episode, which is a defining feature of episodic future thinking (Klein et al., 2004; Suddendorf \& Corballis, 2007; Tulving, 2005). The contribution of self-consciousness and time perspective to future-thinking abilities should therefore not be downplayed.

To conclude, the present results suggest that future thinking involves a collection of processes that are related to different facets of future-event representation (i.e., fluency, specificity, episodic details, and phenomenological characteristics). Executive processes involved in the organization and monitoring of retrieval were correlated with several measures of future thinking, suggesting that they play a general role in accessing and representing futureevent knowledge. The findings further suggest that the 
capacity to generate representations of future events that are infused with sensory details also depends on visualspatial constructive abilities and future time perspective. Finally, the results showed that self-consciousness was related to feelings of experiencing the imagined events, suggesting that the capacity to think about the self and focus attention on one's inner experience may be involved in transforming accessed autobiographical knowledge into the subjective experience of "traveling through time" to preexperience the future.

\section{AUTHOR NOTE}

This research was supported by a grant from the Geneva Neuroscience Center, the French-speaking community of Belgium (ARC, Convention 06/11-340), and the Belgian Fund for Scientific Research (F.R.S.-FNRS). A.D. is a research associate of the F.R.S.-FNRS. The authors thank Sarah Imboden and Federica Salvia for their help in data collection. Correspondence concerning this article should be addressed to A. D'Argembeau, Department of Cognitive Sciences, Centre for Cognitive and Behavioral Neuroscience, University of Liège, Boulevard du Rectorat 3 (B33), 4000 Liège, Belgium (e-mail: a.dargembeau@ulg.ac.be).

\section{REFERENCES}

Addis, D. R., PAn, L., Vu, M. A., Laiser, N., \& Schacter, D. L. (2009). Constructive episodic simulation of the future and the past: Distinct subsystems of a core brain network mediate imagining and remembering. Neuropsychologia, 47, 2222-2238.

Addis, D. R., Sacchetti, D. C., Ally, B. A., Budson, A. E., \& SCHACTER, D. L. (2009). Episodic simulation of future events is impaired in mild Alzheimer's disease. Neuropsychologia, 47, 26602671.

AdDis, D. R., Wong, A. T., \& Schacter, D. L. (2007). Remembering the past and imagining the future: Common and distinct neural substrates during event construction and elaboration. Neuropsychologia, 45, 1363-1377.

Addis, D. R., Wong, A. T., \& Schacter, D. L. (2008). Age-related changes in the episodic simulation of future events. Psychological Science, 19, 33-41.

Apostolidis, T., \& Fieulaine, N. (2004). Validation française de l'échelle de temporalité The Zimbardo Time Perspective Inventory (ZTPI). Revue Européenne de Psychologie Appliquée, 54, 207-217.

Atance, C. M., \& Meltzoff, A. N. (2005). My future self: Young children's ability to anticipate and explain future states. Cognitive Development, 20, 341-361.

Atance, C. M., \& O’Neill, D. K. (2001). Episodic future thinking. Trends in Cognitive Sciences, 5, 533-539.

Benjamini, Y., \& Hochberg, Y. (1995). Controlling the false discovery rate: A practical and powerful approach to multiple testing. Journal of the Royal Statistical Society: Series B, 57, 289-300.

Berntsen, D., \& JACobsen, A. S. (2008). Involuntary (spontaneous) mental time travel into the past and future. Consciousness \& Cognition, 17, 1093-1104.

Botzung, A., Denkova, E., \& Manning, L. (2008). Experiencing past and future personal events: Functional neuroimaging evidence on the neural bases of mental time travel. Brain \& Cognition, 66, 202-212.

Boyer, P. (2008). Evolutionary economics of mental time travel? Trends in Cognitive Sciences, 12, 219-224.

BucKner, R. L., \& CARroll, D. C. (2007). Self-projection and the brain. Trends in Cognitive Sciences, 11, 49-57.

Cardebat, D., Doyon, B., Puel, M., Goulet, P., \& Joanette, Y. (1990). Evocation lexical formelle et sémantique chez les sujets normaux: Performances et dynamique de production en fonction du sexe, de l'âge et du niveau d'étude. Acta Neurologica Belgica, 90, 207217.

Conway, M. A., \& Pleydell-Pearce, C. W. (2000). The construction of autobiographical memories in the self-memory system. Psychological Review, 107, 261-288.

D’Argembeau, A., Raffard, S., \& Van der Linden, M. (2008). Re- membering the past and imagining the future in schizophrenia. Journal of Abnormal Psychology, 117, 247-251.

D'Argembeau, A., Renaud, O., \& Van der Linden, M. (in press). Frequency, characteristics, and functions of future-oriented thoughts in daily life. Applied Cognitive Psychology.

D'Argembeau, A., \& Van der Linden, M. (2004). Phenomenal characteristics associated with projecting oneself back into the past and forward into the future: Influence of valence and temporal distance. Consciousness \& Cognition, 13, 844-858.

D'Argembeau, A., \& VAn Der Linden, M. (2006). Individual differences in the phenomenology of mental time travel: The effect of vivid visual imagery and emotion regulation strategies. Consciousness \& Cognition, 15, 342-350.

D’Argembeau, A., Xue, G., Lu, Z. L., Van der Linden, M., \& Bechara, A. (2008). Neural correlates of envisioning emotional events in the near and far future. NeuroImage, 40, 398-407.

Della Sala, S., Gray, C., Baddeley, A., \& Wilson, L. (1997). The Visual Patterns Test: A new test of short-term visual recall. Feltham, U.K.: Thames Valley Test Company.

Desrochers, A., \& Bergeron, M. (2000). Valeurs de fréquence subjective et d'imagerie pour un échantillon de 1916 substantifs de langue française. Revue Canadienne de Psychologies Expérimentale, 54, 274-325

Gilbert, D. T., \& Wilson, T. D. (2007). Prospection: Experiencing the future. Science, 317, 1351-1354.

Groth-Marnat, G., \& Teal, M. (2000). Block design as a measure of everyday spatial ability: A study of ecological ability. Perceptual \& Motor Skills, 90, 522-526.

Hassabis, D., Kumaran, D., Vann, S. D., \& Maguire, E. A. (2007). Patients with hippocampal amnesia cannot imagine new experiences. Proceedings of the National Academy of Sciences, 104, 1726-1731.

Hassabis, D., \& Maguire, E. A. (2007). Deconstructing episodic memory with construction. Trends in Cognitive Sciences, 11, 299-306.

Henry, J. D., \& Crawford, J. R. (2004). A meta-analytic review of verbal fluency performance in patients with traumatic brain injury. Neuropsychology, 18, 621-628.

Jokinen, H., Kalska, H., Ylikoski, R., Hietanen, M., Mantyla, R., PohjasvaAra, T., ET AL. (2004). Medial temporal lobe atrophy and memory deficits in elderly stroke patients. European Journal of Neurology, 11, 825-832.

Klein, S. B., German, T. P., Cosmides, L., \& Gabriel, R. (2004). A theory of autobiographical memory: Necessary components and disorders resulting from their loss. Social Cognition, 22, 460-490.

Klein, S. B., Loftus, J., \& Kinlstrom, J. F. (2002). Memory and temporal experience: The effects of episodic memory loss on an amnesic patient's ability to remember the past and imagine the future. Social Cognition, 20, 353-379.

Klinger, E., \& Cox, W. M. (1987). Dimensions of thought flow in everyday life. Imagination, Cognition, \& Personality, 7, 105-128.

MacLeod, A. K., \& Byrne, A. (1996). Anxiety, depression, and the anticipation of future positive and negative experiences. Journal of Abnormal Psychology, 105, 286-289.

MacLeod, A. K., \& Conway, C. (2007). Well-being and positive future thinking for self versus others. Cognition \& Emotion, 21, 11141124.

McDermott, K. B., Szpunar, K. K., \& Christ, S. E. (2009). Laboratory-based and autobiographical retrieval tasks differ substantially in their neural substrates. Neuropsychologia, 47, 2290-2298.

New, B., Pallier, C., Brysbaert, M., \& Ferrand, L. (2004). Lexique 2: A new French lexical database. Behavior Research Methods, Instruments, \& Computers, 36, 516-524.

Okuda, J., FujiI, T., Ohtake, H., Tsukiura, T., Tanji, K., Suzuki, K., ET AL. (2003). Thinking of the future and past: The roles of the frontal pole and the medial temporal lobes. NeuroImage, 19, 1369-1380.

Pelletier, L. G., \& Vallerand, R. J. (1990). L'Echelle Révisée de Conscience de Soi: Une traduction et une validation canadiennefrançaise du Revised Self-Consciousness Scale. Revue Canadienne des Sciences du Comportement, 22, 191-206.

Quoidbach, J., Hansenne, M., \& Mottet, C. (2008). Personality and mental time travel: A differential approach to autonoetic consciousness. Consciousness \& Cognition, 17, 1082-1092.

Regard, M., Strauss, E., \& KnapP, P. (1982). Children's production 
of verbal and nonverbal fluency tasks. Perceptual \& Motor Skills, 55, 839-844.

Rubin, D. C. (2006). The basic-systems model of episodic memory. Perspectives on Psychological Science, 1, 277-311.

Rubin, D. C., Schrauf, R. W., \& Greenberg, D. L. (2003). Belief and recollection of autobiographical memories. Memory \& Cognition, 31, 887-901.

Schacter, D. L., \& AdDis, D. R. (2007). The cognitive neuroscience of constructive memory: Remembering the past and imagining the future. Philosophical Transactions of the Royal Society B, 362, 773786.

SCHACTER, D. L., \& AdDIS, D. R. (2009). On the nature of medial temporal lobe contributions to the constructive simulation of future events. Philosophical Transactions of the Royal Society B, 364, 1245-1253.

SCHACTER, D. L., AdDIs, D. R., \& BuCKNER, R. L. (2008). Episodic simulation of future events: Concepts, data, and applications. In A. Kingstone \& M. B. Miller (Eds.), The year in cognitive neuroscience 2008 (Annals of the New York Academy of Sciences, Vol. 1124, pp. 39-60). New York: New York Academy of Sciences.

Scheier, M. F., \& Carver, C. S. (1985). The Self-Consciousness Scale: A revised version for use with general populations. Journal of Applied Social Psychology, 15, 687-699.

Sharot, T., Riccardi, A. M., Raio, C. M., \& Phelps, E. A. (2007). Neural mechanisms mediating optimism bias. Nature, 450, 102-105.

Shelton, J. T., Elliott, E. M., Hill, B. D., Calamia, M. R., \& GouVIER, W. D. (2009). A comparison of laboratory and clinical working memory tests and their prediction of fluid intelligence. Intelligence, 37, 283-293.

Spreng, R. N., \& LeVIne, B. (2006). The temporal distribution of past and future autobiographical events across the lifespan. Memory \& Cognition, 34, 1644-1651.

SudDEndorf, T., \& Busby, J. (2005). Making decisions with the future in mind: Developmental and comparative identification of mental time travel. Learning \& Motivation, 36, 110-125.

Suddendorf, T., \& Corballis, M. C. (1997). Mental time travel and the evolution of the human mind. Genetic, Social, \& General Psychology Monographs, 123, 133-167.

SudDendorf, T., \& Corballis, M. C. (2007). The evolution of foresight: What is mental time travel and is it unique to humans? Behavioral \& Brain Sciences, 30, 299-351.

SzPUNAR, K. K. (2010). Episodic future thought: An emerging concept. Perspectives on Psychological Science, 5, 142-162.

SzPunAR, K. K., \& McDermotT, K. B. (2008). Episodic future thought and its relation to remembering: Evidence from ratings of subjective experience. Consciousness \& Cognition, 17, 330-334.

Szpunar, K. K., Watson, J. M., \& McDermott, K. B. (2007). Neural substrates of envisioning the future. Proceedings of the National Academy of Sciences, 104, 642-647.

Tulving, E. (1985). Memory and consciousness. Canadian Psychologist, 26, 1-12.

Tulving, E. (2005). Episodic memory and autonoesis: Uniquely human? In H. S. Terrace \& J. Metcalfe (Eds.), The missing link in cognition: Origins of self-reflective consciousness (pp. 3-56). Oxford: Oxford University Press.

Uttl, B., Graf, P., \& Richter, L. K. (2002). Verbal paired associates tests limits on validity and reliability. Archives of Clinical Neuropsychology, 17, 567-581.
WeChSLER, D. (2000). Echelle d'intelligence pour adulte (3rd ed.). Paris: Editions du Centre de Psychologie Appliquée.

WeChSLER, D. (2001). Echelle clinique de mémoire (3rd ed.). Paris: Editions du Centre de Psychologie Appliquée.

Wheeler, M. A., Stuss, D. T., \& Tulving, E. (1997). Toward a theory of episodic memory: The frontal lobes and autonoetic consciousness. Psychological Bulletin, 121, 331-354.

Williams, J. M. G., Ellis, N. C., Tyers, C., Healy, H., Rose, G., \& MACLEOD, A. K. (1996). The specificity of autobiographical memory and imageability of the future. Memory \& Cognition, 24, 116-125.

Williams, J. M. G., Healy, H. G., \& Ellis, N. C. (1999). The effect of imageability and predicability of cues in autobiographical memory. Quarterly Journal of Experimental Psychology, 52A, 555-579.

Wilson, B. A., Alderman, N., Burgess, P. W., Emslie, H., \& Evans, J. J. (1996). Behavioural assessment of the dysexecutive syndrome. Bury St Edmunds, U.K.: Thames Valley Test Company.

Zimbardo, P. G., \& Boyd, J. N. (1999). Putting time in perspective: A valid, reliably individual-differences metric. Journal of Personality \& Social Psychology, 77, 1271-1288.

\section{NOTES}

1. An a priori power analysis indicated that a sample size of 95 participants was required to achieve an $80 \%$ statistical power to detect an effect size of $r=.25$ (defined on the basis of previous research looking at the relationship between individual-difference variables and measures of autobiographical memory or future thinking; e.g., D'Argembeau \& Van der Linden, 2006; Rubin, Schrauf, \& Greenberg, 2003) at an alpha level of .05.

2. Unsurprisingly, the number of words reported in the descriptions of events was strongly correlated with the total content scores of the episodic details task: $r=.70$ (95\% CI: .58-.79) and $r=.60$ (95\% CI: $.46-.71)$ for past and future events, respectively. The number of words was also correlated with measures of sensory descriptions: $r=.33(95 \%$ CI: .14-.49) and $r=.28$ (95\% CI: .09-.45), for past and future events, respectively. However, all the correlations involving total content scores and sensory descriptions that are reported in Tables 4 and 5 remained significant after controlling for number of words reported, except the correlation between executive processes and sensory descriptions for past events.

3. In the present study, there was no correlation between our index of visual-spatial processing and the amount of sensory details generated when past events were remembered. That does not mean that visualspatial constructive processes are not engaged when details about past events are remembered, however; when looking specifically at the relationship between performance on the BDT and the number of sensory details generated when past events were remembered, we found that the correlation was close to statistical significance $(r=.18 ; 95 \% \mathrm{CI}:-.02$ to .36). The difference between the past and future might, therefore, be a matter of degree: Visual-spatial constructive processes are probably involved in constructing detailed mental representations of both past and future events (as well as events that are not located in time; Hassabis et al., 2007) but seem to be recruited more intensively for future events.

(Manuscript received July 8, 2009; revision accepted for publication January 10, 2010.) 